\title{
Uso de triancinolona local em uma gata com estenose esofágica
}

\author{
Local triamcinolone in a cat with esophageal stricture \\ Katia Barão Corgozinho', Adriana Neves², Cristiane Belchior ${ }^{3}$, \\ Flávia Toledo ${ }^{4}$, Heloisa Justen Moreira de Souza ${ }^{5}$ \& Aline Santana da Hora ${ }^{6}$
}

\begin{abstract}
RESUMO
A estenose esofágica é uma inflamação no esôfago acometendo as camadas submucosa e muscular com formação de tecido fibroso. O sinal clínico predominante de tal alteração é a regurgitação. O diagnóstico definitivo é realizado através de radiografia contrastada do esôfago e endoscopia. O tratamento inclue os procedimentos conservativos (dilatação através de balão ou por uso de velas) ou cirúrgicos (ressecção e anastomose). O objetivo desse trabalho é relatar o uso de triancinolona local em uma gata com estenose esofágica. Um felino de oito anos de idade, castrada apresentava estenose no esôfago proximal, a causa suspeita foi à ingestão de medicamentos. O tratamento escolhido foi dilatação pelo uso de balão. Após a sétima endoscopia, a gata apresentava regurgitação refratária à dilatação, então foi decidida a administração local de triancinolona. A triancinolona intralesional foi associada com resolução dos sinais nessa paciente. A gata permanece bem seis meses após o corticóide local, apenas regurgita ração de grãos grandes.
\end{abstract}

Descritores: gato, estenose esofágica, triancinolona.

\section{ABSTRACT}

Esophageal stricture is an esophageal inflammation extend through the submucosa into muscularis with formation of fibrous connective tissue. The predominant sign is regurgitation. The definitive diagnosis require demonstration of the stricture by contrast esophagrams and endoscopic examination and the treatment include conservative (dilation by using balloon catheters or bougies) or surgical procedures (resection and anastomosis). The aim of this study is report the use of local triancinolone in a cat with esophageal stricture. The eight-year-old domestic shorthaired neutered cat was present with esophageal stricture in the cervical esophagus, and the suspected cause was administration of medications. The treatment done was dilation by balloon catheters. After the seventh endoscopy, the cat was present with persistent regurgitation refractory to the dilation and we decided to administer local triamcinolne. Intralesional triamcinolone was associated with the resolution of the signs in this cat. She is god six months after the local glucocorticoids and regurgitate when she eats bigger grains.

Key words: cat, esophageal stricture, triamcinolone. 


\section{INTRODUÇÃO}

A esofagite é o resultado de um dano substancial, químico ou físico, à mucosa esofágica [2,4,6,9-12]. Quando a lesão se estende até as camadas submucosa e muscular, ocorre a formação de uma cicatriz circular que compromete o lúmen esofágico levando a estenose [12].

A regurgitação é o principal sinal clínico da estenose esofágica, podendo ocorrer minutos ou horas após a ingestão de água e alimentos [4,6,9,11,12]. A finalidade do tratamento da estenose esofágica é resolver a regurgitação e manter a nutrição e a hidratação adequadas por via oral. A dilatação esofágica por balão ou por velas deve ser realizada se a regurgitação persistir após o manejo clínico [2,3,6,11,12].

Nos casos de estenoses não responsivas à dilatação associada ao uso de corticóides locais, deve-se tentar o tratamento cirúrgico ou uso de próteses, apesar do alto índice de complicação [6,12].

O presente relato descreve uma gata com estenose esofágica não responsiva a prática de dilatação por balão, necessitando da aplicação intralesional de corticóide para sucesso do tratamento.

\section{RELATO DE CASO}

Um felino fêmea, sem raça definida, com oito anos de idade foi atendido devido à dificuldade de deglutição uma semana após a recuperação de um quadro de insuficiência cardíaca congestiva. A gata apresentou dificuldade de engolir, iniciando quadro de regurgitação quando ingeria alimento sólido e dentro de 32 dias não conseguia ingerir a ração úmida. A paciente estava em bom estado geral e demonstrava muito apetite.

Foi realizado exame radiográfico com contraste positivo utilizando sulfato de bário a $10 \%$, misturado à ração úmida, sendo evidenciada estenose esofágica no terço proximal do esôfago (Figura 1). Após o diagnóstico radiográfico foi realizada endoscopia que confirmou a presença de estenose esofágica com aproximadamente $3 \mathrm{~mm}$ de diâmetro. Durante a endoscopia optou-se pela dilatação da estenose cicatricial através da técnica de balonamento. Devido ao diâmetro reduzido da estenose utilizou-se inicialmente um cateter de Folley com balão de $3 \mathrm{~mL}$. Após a ruptura da cicatriz e passagem do fibroscópio através da estenose, foi realizada a gastrostomia endoscópica percutânea com colocação de cateter para alimentação

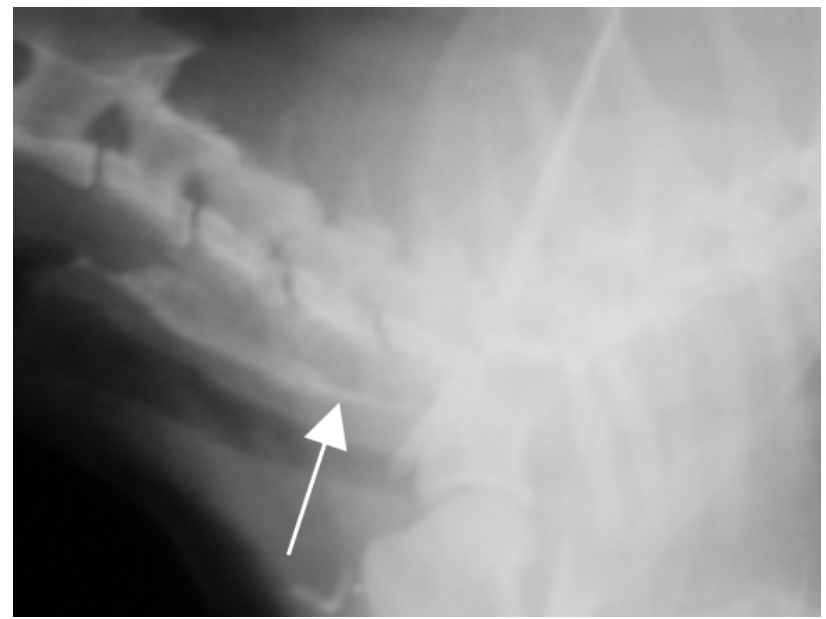

Figura 1. Radiografia contrastada evidenciando diminuição da luz esofágica na região cervical (seta branca).

enteral. A gata foi medicada com ampicilina na dose de $40 \mathrm{mg} / \mathrm{Kg}$ a cada oito horas durante 15 dias e predinsolona $0,5 \mathrm{mg} / \mathrm{kg}$ a cada 12 horas por 15 dias. Foram realizadas mais quatro endoscopias com o intervalo de sete dias entre elas. Nesses procedimentos foram utilizados cateteres de Folley de 5 e $10 \mathrm{~mL}$ e um balão de angioplastia para as dilatações. $\mathrm{O}$ paciente iniciou a ingestão de raça úmida após dez dias da primeira dilatação e de ração seca 42 dias após. As quatro avaliações endoscópicas revelaram recidiva da estenose esofágica, porém sem dificuldade na dilatação. Em decorrência da alteração cardíaca e prostração da gata após a quinta endoscopia, os procedimentos foram suspensos, uma vez que o animal conseguia se alimentar com ração seca.

A gata voltou a apresentar regurgitação e 30 dias após a quinta endoscopia não conseguia ingerir ração úmida. Foram realizadas mais cinco endoscopias com dilatação. Devido à recidiva da estenose, durante o oitavo procedimento optou-se pela injeção local de $40 \mathrm{mg}$ de acetato de triancinolona na estenose, $20 \mathrm{mg}$ da medicação foram aplicados antes da dilatação e $20 \mathrm{mg}$ após a dilatação. A estenose foi dividida em quatro quadrantes, sendo aplicados $5 \mathrm{mg}$ em cada quadrante. Após uma semana foi realizada nova avaliação endoscópica que revelou maior abertura do lúmen esofágico e uma lesão ulcerativa. A gata foi medicada com antibioticoterapia (ampicilina) e protetor de mucosa (sucralfato). $\mathrm{O}$ décimo e último procedimento endoscópico evidenciou melhora acentuada da estenose, permitindo a passagem sem dificuldade do fibroscópio e a lesão ulcerativa havia diminuído. A paciente voltou a comer ração seca e úmida 
sem apresentar regurgitação. Seis meses após o término do tratamento o animal regurgita esporadicamente quando come ração seca de grãos grandes.

\section{DISCUSSÃO}

A estenose pode se desenvolver em qualquer segmento esofágico e dependerá da causa. Estenoses secundárias causadas por refluxo gástrico ocorrem comumente no terço médio e distal do esôfago e aquelas decorrentes da administração de cápsulas ou comprimidos acometem com maior frequiência o esôfago cervical [5,10-12]. A gata desse relato apresentava estenose esofágica no terço proximal levando a suspeita de administração medicamentosa como a causa da lesão. Esse felino recebeu vários fármacos e dentre eles o antibiótico clindamicina em forma de cápsula, não sendo administrada água após qualquer medicação. Isso leva a suspeita de retenção da cápsula no esôfago permitindo a base medicamentosa lesar a mucosa esofágica, assim como em um estudo onde foi relatado que as cápsulas administradas sem acréscimo de água ficaram retidas no esôfago por mais de 300 segundos, principalmente no segmento esofágico médio [13]. Em outro estudo, foi observado, também, que o trânsito de cápsulas pelo esôfago em gatos pode ser demorado ou até pode ocorrer à retenção das mesmas [5].

Nesse presente relato, corpo estranho, ingestão de substância cáustica e vômitos foram eliminados como possíveis causas na formação de estenose, assim como foi observado em outro relato [9]. O histórico da administração de clindamicina 17 dias antes das manifestações clínicas de estenose esofágica, sugere a administração do medicamento como fator causal de tal alteração, pois os sinais clínicos geralmente se desenvolvem uma a três semanas após a causa primária $[4,6,9,11]$.
Embora o uso sistêmico de corticóides não seja comprovadamente benéfico para manejo de esofagite e estenose esofágica em humanos, sua aplicação local tem sido estudada. A aplicação local de triancinolona, não foi associada à resolução da estenose, mas a completa resolução dos sinais clínicos. Esse fármaco aplicado intralesional impede a contração da estenose após sua dilatação [3]. A dose usada em cães varia de 20 a $40 \mathrm{mg}$, dividindo a estenose em quatro quadrantes aplicando um quarto da dose em cada quadrante [1]. O uso de corticóide sistêmico na gata desse relato não ajudou na redução da contração da ferida ocorrendo nova estenose, necessitando ser aplicado na lesão. Esse uso intralesional foi efetivo nesse felino com a mesma dose usada para cães. Até então, a gata havia realizado sete endoscopias e sempre ocorria o retorno da estenose, até ser utilizado o acetato de triancinolona local. Essa aplicação foi importante considerando que a gata era um paciente crítico e não poderia ficar sendo submetida a repetidos procedimentos endoscópicos.

O tratamento local melhorou a qualidade de vida do animal e o seu estado nutricional, sendo observado o mesmo por outros pesquisadores [8] que trataram de dois pacientes humanos com estenose esofágica refratária à dilatação por balão utilizando triancinolona local diminuindo a frequiência entre as dilatações. A melhora considerável da estenose após o uso de triancinolona foi observada também por outros autores que trataram de seis cães com estenose esofágica por substância cáustica [1].

Conclui-se que a injeção de corticosteróide local é segura, efetiva e barata para o tratamento de estenoses esofágicas refratárias à dilatação. Por ser um dos poucos casos relatados em animais de pequeno porte, mais estudos devem ser realizados para avaliação da eficácia e dos efeitos colaterais dessa medicação em cães e gatos.

\section{REFERÊNCIAS}

1 Ashcraft K.W. \& Holder T.M. 1969. The experimental treatment of esophageal strictures by intralesional steroid injections. Journal of Thoracic and Cardiovascular Surgery. 58: 685-693.

2 Burk R.L., Zawie D.A. \& Garvey M.S. 1987. Ballon cathter dilation of intramural esophageal strictures in the dog and cat: a description of the procedure and a report of six cases. Seminars in Veterinary Medicine and Surgery (Small Animal). 2: 241-247.

3 Gandhi R.P., Cooper A. \& Barlow B.A. 1989. Successful management of esophageal strictures without resection or replacement. Journal of Pediatric Surgery. 24: 745-750.

4 German A.J., Cannon M.J., Dye C., Booth M.J., Pearson G.R., Reay C.A. \& Jones T.J.G. 2005. Oesophageal strictures in cats associated with doxycycline therapy. Journal of Feline Medicine and Surgery. 7: 33-41.

5 Graham J.P., Lipman A.H. Newell S. M. \& Roberts G.D. 2000. Esophageal transit of capsules in clinically normal cats. American Journal of Veterinary Research. 61: 655-657. 
6 Gualtieri M. 2001. Esophagoscopy. The Veterinary Clinics of North America: Small Animal Practice. 31: 605-630.

7 Haral B.H., Johnson S.E. \& Sherding R.G. 1995. Endoscopically guided ballon dilatation of benign esophageal strictures in 6 cats and 7 dogs. Journal of Veterinary Internal Medicine. 9: 332-335.

8 Kirsch M., Blue M., Desai R.K. \& Sivak M.V. 1991. Intralesional steroid injections for peptic esophageal strictures. Gastrointestinal Endoscopy. 37: 180-182.

9 McGrotty Y.L. \& Knottenbelt C.M. 2002. Oesophageal stricture in a cat due to oral administration of tetracyclines. Journal of Small Animal Practice. 43: 221-223.

10 Melendez L.D., Twedt D.C. \& Wright M. 2000. Suspected doxycycline- induced esophagitis with esophageal stricture formation in three cats. Feline Practice. 28: 10-12.

11 Moraitou K.K.A., Rallis T.S., Prassinos N.N. \& Galatos A.D. 2002. Benign esophageal stricture in the dog and cat: a restropective study of 20 cases. The Canadian Journal of Veterinary Research. 66: 55-59.

12 Sellon R.K. \& Willard M.D. 2003. Esophagitis and esophageal strictures. The Veterinary Clinics of North America: Small Animal Practice. 33: 945-967.

13 Westfall D.S., Twedt D.C., Steyn P.F., Oberhauser E.B. \& VanCleave J.W. 2001. Evaluation of esophageal transit of tablets and capsules in 30 cats. Journal of Veterinary Internal Medicine. 15: 467-470. 